\title{
A Reputation Revision Method Based on the Credibility for Cloud Services
}

\author{
Xiaogang Cai ${ }^{1}$, Mingchuan Zhang ${ }^{1 *}$, Jiankun $\mathrm{Hu}^{2}$, Qingtao $\mathrm{Wu}^{1,2}$ \\ ${ }^{1}$ Information Engineering College, Henan University of Science and Technology, \\ Luoyang 471023, Henan Province, China \\ ${ }^{2}$ School of Engineering and Information Technology, \\ University of New South Wales, Canberra ACT 2600, Australia \\ cxg1666@163.com, zhang_mch@haust.edu.cn*,j.hu@adfa.edu.au, lywxr2007@haust.edu.cn
}

Keywords: cloud service, reputation revision, rating credibility, participation degree, incentive. Abstract. The reputation system has obvious advantages over the traditional technology in solving the trust problem of cloud services. However, there are some problems in reputation system, such as user evaluation lacks enthusiasm, false evaluation and so on, which affect the availability of reputation system seriously. This paper proposes an evaluate revision reputation method based on credibility of nodes. On the basis of the distributed computing of cloud service reputation, it not only modifies reputation value to increase the accuracy of reputation result by calculating reputation of each node, but also introduces the incentive mechanism to improve the enthusiasm of the evaluation cloud user nodes. The simulation results verify the feasibility and effectiveness of the method.

\section{Introduction}

The research shows that the reputation evaluation system is helpful to establish the online reputation. The reputation has been widely used in the business evaluation of online trade, the upload node selection of $p 2 p$ file transfer, the grid computing and other fields ${ }^{[1,2]}$. For the open, dynamic cloud service environment, the introduction of reputation system provides the basis for the users to obtain accurate and reliable cloud services. How to build an effective reputation evaluation model for cloud services has become a research hotspot ${ }^{[3,4]}$.

Paper [5] presented a reputation measurement model, which used fuzzy logic to calculate the reputation value of cloud services. Paper [6] designed a kind of service selection method, which is based on a reputation revision method. Paper [7] proposed a detection technology for the reputation of cloud services, so that users can effectively identify the trusted cloud services. Paper [8] presented a new method which is used as a reference to identify and filter out the inaccurate ratings by evaluating the mean value of similarity with the priori knowledge. Paper [9] proposed a new model based on $D-S$ evidence theory, which uses the fuzzy theory to deal with the customer's satisfaction with the related service. Paper [10] proposed a user experience quality model based on the Markoff and an evaluating method for the random model.

In reputation evaluation for the cloud services, people pay more attention to the cloud service provider, ignored the user reputation. This makes the user evaluation lack of credibility. In this case, some users don't actively participate in the evaluation, thus the difficulty of acquiring the evaluation information has been greatly increased, and finally affected the availability of reputation system.

According to the above problem, this paper proposes a reputation revision method based on credibility of user nodes. Measure reputation of each node according to the direct experience between the evaluated nodes and the cloud service providers. Credibility of the high and low will directly affect the weight of calculating evaluate reputation of cloud services The method also introduces the incentive mechanism. The changes in participation degree for each evaluation node will affect its credibility to some extent, and then affect the quality of recommendation cloud services the user will obtain. Hence arouse the enthusiasm of user nodes to evaluate honestly. 


\section{Reputation Evaluation and Revision Model for Cloud Services}

As is shown in Fig.1. The model manages the reputation of cloud services, service providers and users by the combination of reputation computing, reputation revision and evaluation incentive, which made the distributed reputation evaluation model more credible. To encourage cloud service users to evaluate actively, we introduced participation degree. Then we revise the reputation computing value based on the dynamic credibility of the users. Finally, the reputation center will recommend different cloud services for users based on their credibility and preference.

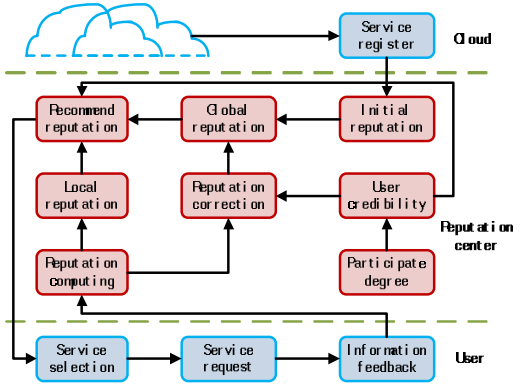

Fig.1 Reputation evaluation model for cloud services

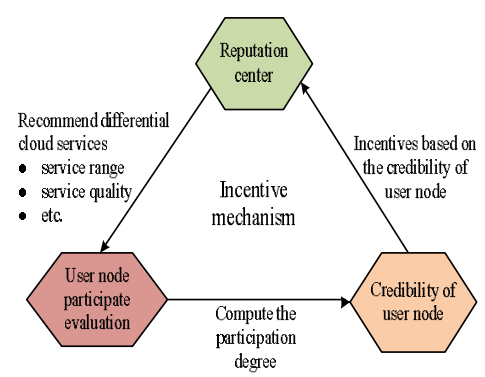

Fig. 2 the incentive model for republican evaluation

Definition 1. Rating Credibility $(R C)$ : it reflects the authenticity or accuracy of the evaluation submitted by the user node. The higher the value of reputation, the more creditable the evaluation.

Definition 2. Participation Degree $(P d)$ : we define the $P d$ as follows:

$$
P d= \begin{cases}\frac{R}{E}, & \text { if } E \neq 0 \\ 0.5, & \text { else }\end{cases}
$$

As is show in Eq 1, $R$ is the number of evaluation and $E$ is the total number of cloud services users got in a period of time. The initial value of participation degree is 0.5 and the range is: $0 \leq P d \leq 1$.

\section{Reputation Evaluation and Revision Policy}

Reputation Computing for Cloud Services. The reputation computing for cloud services includes global reputation computing and local reputation computing. $G R$ is the global reputation stored in the reputation center, $L R$ is the local reputation stored in the user's local storage. $\left(S I D, \mathrm{~S}_{i}, F_{i}\right)$ is defined as the index for the registered cloud service. SID is a specific service, the $S_{i}$ is the number of times the index $i$ of this service has reached the specified level and $F_{i}$ is the number of times the index $i$ of this service has not reached the specified level. In the evaluation, the satisfaction is marked as $s_{i}=1$, while the not satisfaction is marked as $f_{i}=1$. And $s_{i}+f_{i}=1$.

$$
L R(\operatorname{SID})=F_{o}\left(q_{1}, q_{2}, \ldots, q_{n}\right)+F_{s}(\text { user })
$$

The $F_{o}$ is a function which integrated related indicators $q_{i}$ for $Q o S$. The $F_{s}$ is a function to handle user satisfaction. As is shown in Eq 3.

$$
F_{o}\left(q_{1}, q_{2}, \ldots, q_{n}\right)=\frac{1}{n} \sum_{i=1}^{n}\left(\frac{S_{i}+s_{i}}{S_{i}+F_{i}+1}\right)
$$

The $G R$ are calculated by collecting all the evaluation of the same service. In order to reflect the timeliness of reputation and reduce the impact of historical reputation, we introduce the exponential decay factor to increase the influence of real time service. As is shown in Eq 4. 


$$
G R=G R^{\prime} \times e^{-\alpha \Delta t}+\frac{1}{n} \sum_{i=1}^{n} f\left(L R_{i}\right)
$$

Reputation Revision Based on the Credibility of Users. We revised the reputation evaluation by introducing the credibility of the evaluation node. The function $f$ is shown in $\mathrm{Eq} 5$.

$$
f\left(L R_{i}\right)=F_{o}\left(q_{1}, q_{2}, \mathrm{~L}, q_{n}\right)+F_{s}(\text { user }) \times R C_{i}
$$

$(U I D, R C)$ is set as an attribute identifier used to associate a specific user's value of rating credibility. The user's rating credibility is usually influenced by three factors: 1 ). subjective and objective rating similarity; 2). subjective and majority rating similarity; 3 ). subjective rating stability.

The Subjective and Objective Rating Similarity (SORS). The subjective evaluation and objective evaluation measure the performance of service from two angles, and the two groups should be consistent or have high similarity.

We regard the subjective evaluation sequence $S r=\left(s r_{1}, s r_{2}, \mathrm{~L}, s r_{n}\right)$ and objective evaluation sequence $O r=\left(o r_{1}, o r_{2}, \mathrm{~L}, o r_{n}\right)$ as two points in n-dimensional apace. By calculating the Euclidean distance of the two points, we can measure the subjective and objective rating similarity. The smaller the distance, the greater the similarity and the higher the credibility of subjective evaluation. As is shown in the Eq 6.

$$
\text { SORS }=\sqrt{\sum_{1}^{n}\left(s r_{i}-o r_{i}\right)^{2}}
$$

The subjective and majority rating similarity (SMRS). It is generally considered that the subjective evaluation of most user nodes in the evaluation system is reasonable and reliable. Based on this, we use the $k$-means algorithm to cluster the evaluation information $R=\left\{r_{i} \mid i=1,2, \mathrm{~L}, n\right\}$ in a period of time. $C_{j}(j=1,2, \mathrm{~L}, k)$ indicates $k$ class of clustering, $c_{j}(j=1,2, \mathrm{~L}, k)$ indicates the initial clustering center. We choose the center of the maximum cluster as the majority rating $(M R)$ value of $R: M R=$ center $\left(\max \left(C_{j}\right)\right) . M R$ is used as a reference, and the Euclidean distance between the user subjective evaluation sequence $S r$ and $M R$ is used to calculate $S M R S$. As is shown in the Eq 7.

$$
S M R S=\sqrt{\sum_{1}^{n}\left(s r_{i}-m r_{i}\right)^{2}}
$$

The smaller the value of SMRS, the higher the credibility of users.

The subjective rating stability (SRS). We use the discrete degree of subjective evaluation sequence to illustrate the stability of the user node. It can reflect the evaluation credibility of user from one aspect. As is shown in Eq 8.

$$
S R S=\sqrt{\frac{1}{n} \sum_{i=1}^{n}\left(s r_{i}-\overline{s r}\right)^{2}}
$$

The $S r_{i}$ is the user's subjective evaluation sequence value, the $\overline{s r}$ is the mean of the evaluation. The smaller the $S R S$ which explains that user evaluation is relatively concentrated in surrounding the $\overline{s r}$, so the evaluation value is relatively stable, the user evaluation is rational and credible.

In order to encourage users to actively participate in the evaluation, we introduce the $P d$ to describe the degree of participation in the evaluation, which is set as a factor in the calculation of the reputation evaluation. As is shown in Eq 9

$$
R C=\frac{1}{(\alpha \cdot S O R S+\beta \cdot S M R S+\varepsilon \cdot S R S)} \times(P d+\delta)
$$


In Eq 9, the $\delta$ is nonzero adjustment factors and $0<\delta \leq 1,0<(\alpha, \beta, \varepsilon)<1, \alpha+\beta+\varepsilon=1$.

Incentive Mechanism for Republican Evaluation. As is shown in Fig.2. After having made a comment, we will get the participation degree of user in the period of time by calculating the user $P d$. As a factor, the user $P d$ will be involved in the calculation of user credibility, which will affect the rating credibility of user. The reputation center determined the range and quality of recommend service based on the user $R C$ before recommending cloud services. Therefore the $P d$ will affect the quality of recommended services the user can get. That is, with low participation, users may eventually get poor quality cloud services. The mechanism allows users to actively participate in the evaluation in order to obtain a high quality of cloud services, thus completing the incentive.

1) Reputation center: the reputation center not only can calculate the exact value of the cloud service, but also provide the different recommended cloud services according to the user node's credibility. The recommended services the user node will get which has the higher credibility have many advantages, such as the range is wider, the quality is higher, the response to the request of the time is faster and so on.

2) User $R C$ : on the one hand, the credibility of the user node participates in the calculation of the reputation evaluation as the weight of its evaluation. On the other hand, it also provides a reference for the service recommendation.

3) User node participated in evaluation: in addition to subjective evaluation, the $R C$ of user node still is related to the $P d$ of user node. It is important for the $R C$ of user node whether the user node positively participate in the evaluation or not.

\section{Experiments and analysis}

Reputation Evaluation Simulation Experiment. In the experiment, this system consists of 100 cloud services and 60 different types of users. The reputation evaluation of cloud services is divided into four categories: normal, good, bad, and excellent. The evaluation of users are divided into three categories: preference evaluation, objective evaluation and deviation evaluation. In order to reflect the user's type, the true quality of cloud services is known for each user in the process of evaluation.

In the experiments, we validated two hypotheses: 1) Calculating the user's credibility of evaluation can improve the accuracy of the results of reputation computing. 2) Introducing the evaluation incentive model can effectively improve the enthusiasm of the user to participate in the evaluation.

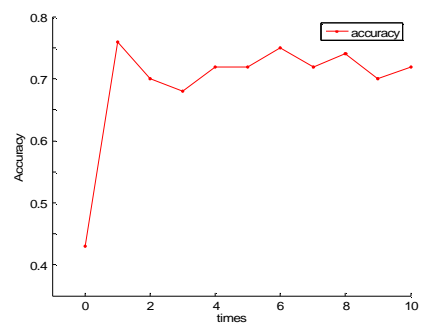

Fig. 3 the accuracy of reputation evaluation For cloud service

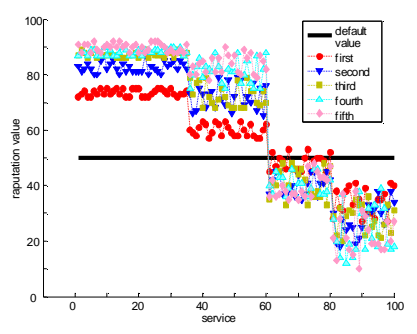

Fig.4 the reputation value of each service after five round of the evaluation

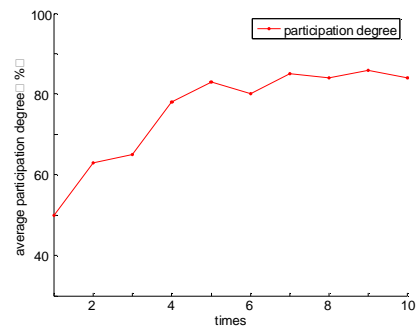

Fig.5 the user participation degree after ten round of reputation evaluation

As is shown in Fig.3, the initial reputation value 0.43 is often not able to characterize the true quality level. The accuracy of global reputation value remain at the level of $75 \%$ with continuous change after a round of reputation evaluation. This proves that the process of reputation calculation is dynamic and tends to be objective, and it can describe the quality level of service after a lot of reputation accumulation. However, due to the 60 users and 100 service users are fixed, the reputation value will tend to be stable in the absence of new users to join the evaluation system.

Fig. 4 shows the distribution of reputation value for cloud service after five round of reputation evaluation. The initial value is set as 0.5 . After a period of accumulation, it can clearly distinguish different cloud service quality level. The service has roughly four levels, but inaccurate reputation value is still exist, which is affected by the existence of the dishonest evaluation and subjective preferences of 
users. The deviation of reputation for cloud service will gradually decrease when the system has sufficient number of users.

Simulation Test of Incentive Mechanism for Reputation Evaluation. In order to verify the validity of the proposed evaluation incentive model, we have isolated the incentive mechanism from the system, in accordance with the following rules:

1) The rating credibility of user is directly related to the participation degree: $R C_{\text {i-new }}=R C_{i-\text { old }} \times\left(P d_{i-n e w}+\delta\right)$ the $0 \leq \delta \leq 1$ is the adjust factor. The initial user $P d$ is defined as 0.5 , $\delta=0.2$. Users will actively participate in the evaluation in order to maintain or want to obtain higher credibility.2) We introduce the exponential decay factor to reflect the influence of real time

participation degree: $\left.P d_{i-n e w}=P d_{i-o l d} \times e^{-\alpha \Delta t}+\frac{R}{E} .3\right)$ We count the average participation of all users after each round of evaluation: $\overline{P d}=\frac{1}{n} \sum_{i=1}^{n} P d_{i-n e w}$, the $i$ is the number of user received cloud services.4) We set that the ratio of user who want to maintain their original reputation is $\lambda$ and the ratio of user who want the higher reputation is $\varepsilon$. The $\varepsilon$ and $\lambda$ randomly generated within range: $0 \leq \lambda \leq 1,0 \leq \varepsilon \leq 1$.

As is shown in Fig.5, the user's initial average participation degree is 0.5 . Users will actively participate in the evaluation in order to get high credibility, so the average degree of participation is increasing after each round. The increasing tends to be gentle when participation degree reached $80 \%$. This is because the adjust factor $\delta=0.2$ and $\lambda$ is far greater than $\varepsilon$.

\section{Conclusions}

The emergence of massive cloud services forms a challenge for the user to identify and select the cloud services with high quality. 1) This paper proposes a reputation management mechanism based on the credibility of the user, using reputation degree of the cloud services to provide some references for the service selection of users. 2) Introducing the idea of the incentive mechanism to provide mentality for solving the negative pretermission in the evaluation participation. 3) The method we have proposed is effective, but still has some problems to improve. Next step, we would consider the influence of complexity which introduced reputation degree on the whole reputation mechanism, and may improve the efficiency of the system.

\section{Acknowledgement}

This work is partially supported by the National Natural Science Foundation of China (NSFC) under Grant no.61370221 and U1404611, in part by Program for Science \& Technology Innovative Research Team in University of Henan Province under Grant no. 14IRTSTHN021.

\section{References}

[1] Yu Jin, Minzhi Gu. A survey of key problems of trust-based on reputation in peer-to-peer systems, J. Journal of Chinese Computer Systems, 2008, 28(9). In Chinese.

[2] Yongrui Cui, Mingchu Li, Yang Xiang. A QoS- based fine- grained reputation system in the grid environment, J. Concurrency \& Computation Practice \& Experience, 2012, 24(17):1990-2006.

[3] Achim O M, Pop F, Cristea V. Reputation based selection for services in cloud environments, C. Network-Based Information Systems (NBiS), 2011 14th International Conference on. IEEE, 2011: 268-273.

[4] Itani W, Ghali C, Kayssi A I, et al. Accountable reputation ranking schemes for service providers in cloud computing, C. CLOSER 2011-Proceedings of the 1st International Conference on Cloud Computing and Services Science, Noordwijkerhout, Netherlands, 7-9 May, 2011. 2011: 49-55. 
[5] Shangguang Wang, Jie Wei, Lei Sun. Reputation measurement of cloud services based on unstable feedback ratings, C. Parallel and Distributed Systems (ICPADS), 2013 International Conference on. IEEE, 2013: 474-479.

[6] Shenghui Zhao, Guoxin Wu, Guilin Chen, Haibao Chen. Reputation-aware service selection based on QOS similarity, J. Journal of Networks. 2011, 6(7): 950-957.

[7] Noor T H, Sheng Q Z, Alfazi A. Reputation attacks detection for effective trust assessment among cloud services, C. Trust, Security and Privacy in Computing and Communications (TrustCom), 2013 12th IEEE International Conference on. IEEE, 2013: 469-476.

[8] Qingtao Wu, Xulong Zhang, Mingchuan Zhang. Reputation revision method for selecting cloud services based on prior knowledge and a market mechanism, J. The Scientific World Journal, 2014, 2014(2):617087-617087.

[9] Ping Wang. Managing service reputation with vague sets, C. e-Business Engineering (ICEBE), 2012 IEEE Ninth International Conference on. IEEE, 2012: 103-110.

[10] Chuang Lin, Jie Hu, Zhenxiang Kong. Survey on models and evaluation of quality of experience, J. Chinese journal of computers, 2012, 35(1): 1-15. In Chinese. 